\title{
Chemotherapy-induced complications in patients with lung cancer: An evaluation by pharmacists
}

\author{
ASUKA ISHIKAWA ${ }^{1}$, GEN OHARA ${ }^{2}$, KENSUKE NAKAZAWA ${ }^{2}$, TOMOHIRO TAMURA ${ }^{2}$, SHINYA SATO $^{2}$, \\ KATSUNORI KAGOHASHI ${ }^{2}$, KOICHI KURISHIMA ${ }^{2}$, YOKO ITO ${ }^{1}$ and HIROAKI SATOH ${ }^{2}$ \\ ${ }^{1}$ Division of Pharmacy, Mito Kyodo General Hospital; ${ }^{2}$ Division of Respiratory Medicine, \\ Mito Medical Center, University of Tsukuba, Mito, Ibaraki 310-0015, Japan
}

Received August 8, 2012; Accepted September 24, 2012

DOI: $10.3892 /$ mco.2012.33

\begin{abstract}
In lung cancer patients, chemotherapy-induced complications are considered to be distressing reactions even in the era of new antiemetics, such as aprepitant. The aim of this study was to evaluate the incidence of such complications. This prospective observational study was performed in our institution between 2011 and 2012. Certain complications including nausea, vomiting, appetite, stomatitis, constipation, diarrhea and dysesthesia, on days 1-7 were evaluated by pharmacists. The questionnaires and diaries of chemotherapy-induced complications were evaluated in the 31 patients included in the study. The majority of the enrolled patients were male $(81 \%)$. Six $(19 \%)$ patients were administered cis-diamminedichloroplatinum(II) (CDDP)-, 21 (69\%) chemotherapy by carboplatin (CBDCA)- and 4 (13\%) non-platinum regimen chemotherapies. Ten $(32.3 \%)$ of the 31 patients exhibited nausea but only $3(9.7 \%)$ of them experienced vomiting. On days 5-6, 23.8 and 9.5\%, respectively, of patients treated with CDDP-regimens had nausea and vomiting. Three of the other most common complications were constipation, general fatigue and appetite loss. The incidence of these complications was 77.4, 71.0 and $67.7 \%$, respectively. Even in the era of new antiemetics, CDDP-regimen chemotherapy-induced nausea and vomiting as well as constipation; general fatigue and appetite loss continue to be problems. A better appreciation of the incidence of these chemotherapy-related complications by medical oncologists and medical staff is essential for their adequate control.
\end{abstract}

\footnotetext{
Correspondence to: Professor Hiroaki Satoh, Division of Respiratory Medicine, Mito Medical Center, University of Tsukuba, Miya-machi 3-2-7, Mito, Ibaraki 310-0015, Japan

E-mail: hirosato@md.tsukuba.ac.jp
}

Key words: chemotherapy-induced complications, lung cancer, pharmacists

\section{Introduction}

Chemotherapy-induced complications are distressing reactions (1). The incidence, prevalence and severity of the complications are associated with several factors. Notably, the emetic risk of the chemotherapy is correlated with the specific drug, dose, schedule and route of administration, as well as with patient variables. Among the complications, chemotherapy-induced nausea and vomiting (CINV) has been reduced by metoclopramide, serotonin (5-HT3) antagonists and corticosteroids without additional severe toxicity (2). Nevertheless, the control of acute and delayed CINV with these antiemetic therapies has not been proven to be sufficient, especially in patients receiving highly (HEC) and moderately emetogenic chemotherapy (MEC). As a result, more effective antiemetic treatments are still required $(3,4)$. This has led to the development of a new class of antiemetic agents, such as aprepitant, an antagonist of the neurokinin-1 (NK-1) receptor. The addition of aprepitant to 5-HT3 receptor antagonist and dexamethasone in cisplatin-based chemotherapy markedly reduces acute and delayed emesis (5). This three-drug combination has also been investigated, with favorable results, in patients receiving a combination of an anthracycline and cyclophosphamide-based regimen, and these studies were funded by pharmaceutical companies $(6,7)$.

The aim of this study was to determine the incidence of chemotherapy-induced complications. Pharmacists, who had no conflicts of interest with any pharmaceutical companies, completed a questionnaire regarding their perceptions of the incidence of complications.

\section{Materials and methods}

Study description. This prospective observational study was conducted at Mito Kyodo General Hospital, Mito, Ibaraki, Japan, between 2011 and 2012. A survey asking pharmacists to predict the incidence of complications including CINV and appetite loss following HEC or MEC was used. Patients administered HEC or MEC regimens at our Institution were recruited. Eligible patients were adults, administered HEC or MEC [emetic levels 3-5 of chemotherapy as defined by Hesketh et al (5)] (Table I). Patients were ineligible in case they were unable to complete the questionnaires. Written and signed 
informed consent was obtained from the patients. The protocol was approved by the Hospital's Ethics Committee.

Antiemetic therapy. Patients administered antiemetic treatment with a three-drug regimen that included aprepitant $(125 \mathrm{mg} /$ day on day $1,80 \mathrm{mg} /$ day on days $2-3)$, dexamethasone $(6.6 \mathrm{mg} /$ day drip infusion on days 1-3) and granisetron (3 mg drip infusion on day 1). Rescue therapy was administered when necessary. Cisplatin-based regimens were considered HEC regimens, carboplatin-based regimens were evaluated as MEC and non-platinum regimens were considered MEC regimens.

Study measurements. Pharmacists completed a questionnaire regarding their perceptions of the incidence of complications including CINV in their own practices subsequent to chemotherapy, despite the use of adequate antiemetic therapy.

Based on previous studies (8-11), seven complications on days 1-7 were evaluated in this study. The extent of the complications were: nausea (none, slightly but no effect on eating, hard to eat, cannot eat and drink), vomiting (number of vomiting episodes per day), appetite (no change, slightly decreased, half decreased, considerably decreased), stomatitis (none, slightly but no effect on eating, effect on eating, hard to eat, cannot eat), constipation (none, slightly but without medication, slightly with medication, moderate with medication, with abdominal pain), diarrhea (none, slightly but without medication, slightly with medication, moderate with medication, with abdominal pain), dysesthesia (none, slightly, moderately, hard to work) and general fatigue (none, slightly, moderately, hard, severe). Patients were instructed to use a diary to report each episode of complication. In this study, we evaluated each complication when present, whether a modest or considerable degree was observed.

\section{Results}

The total number of patients who completed the questionnaires and diaries was 31 . The patient characteristics are shown in Table I. The majority of enrolled patients were male (80.6\%). The median age of the 31 patients was 69 years (range, 60-82 years). Six (19.4\%) patients were administered CDDP-, $21(67.7 \%)$ CBDCA- and 4 (12.9\%) non-platinum regimens. There were no severe complications requiring additional medication.

Control of nausea and emesis. The percentage of patients reporting nausea/day following chemotherapy treatment was recorded (Fig. 1). Regarding nausea, 10 (32.3\%) of the 31 patients exhibited the complication between days 1-7. In patients treated with CDDP-regimens, the incidence of nausea on each day was: day $1,4.8 \%$; day $2,4.8 \%$; day $3,14.3 \%$; day 4 , $23.8 \%$; day $5,23.8 \%$; day $6,19.0 \%$ and day $7,14.3 \%$. In patients treated with CBDCA-regimens, the incidence of nausea on each day was: days $1-4,0 \%$; day $5,16.7 \%$ and day $6-7,0 \%$. In patients treated without platinum-regimens, the incidence of nausea on days 1-7 was $0 \%$.

The percentage of patients reporting vomiting/day following chemotherapy treatment was recorded (Fig. 2). Vomiting was observed in $3(9.7 \%)$ of the 31 patients (Table I). In patients treated with CDDP-regimens, 9.5\% exhibited
Table I. Characteristics of patients with lung cancer.

\begin{tabular}{lr}
\hline Characteristics & Value \\
\hline Age (years), median (range) & $69(60-82)$ \\
Gender, n (\%) & \\
Male & $25(80.6)$ \\
Female & $6(19.4)$ \\
Treatment regimens, n (\%) & \\
CDDP-regimens & $6(19.4)$ \\
CBDCA-regimens & $21(67.7)$ \\
Non-platinum regimens & $4(12.9)$ \\
Complications, n $(\%)$ & \\
Nausea & $10(32.3)$ \\
Vomiting & $3(9.7)$ \\
Constipation & $24(77.4)$ \\
General fatigue & $22(71.0)$ \\
Appetite loss & $21(67.7)$ \\
Dysesthesia & $6(19.4)$ \\
Stomatitis & $4(12.9)$ \\
Diarrhea & $4(12.9)$ \\
\end{tabular}

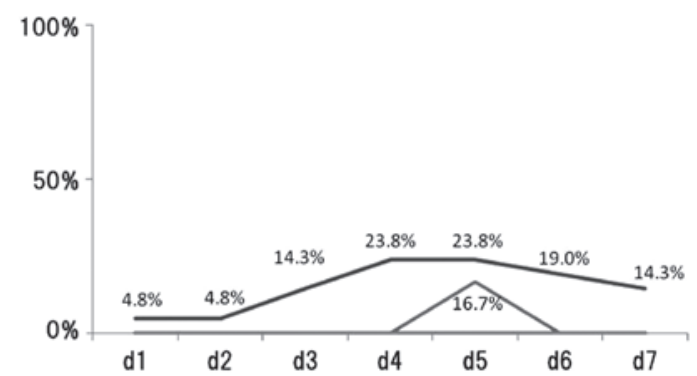

Figure 1. Percentage of patients reporting nausea/day after chemotherapy treatment is shown. Upper line indicates the incidence of nausea in patients administered CDDP-regimens. Middle line indicates the incidence of nausea in patients administered CBDCA-regimens. Lower line indicates the incidence of nausea in patients administered the non-platinum regimen.

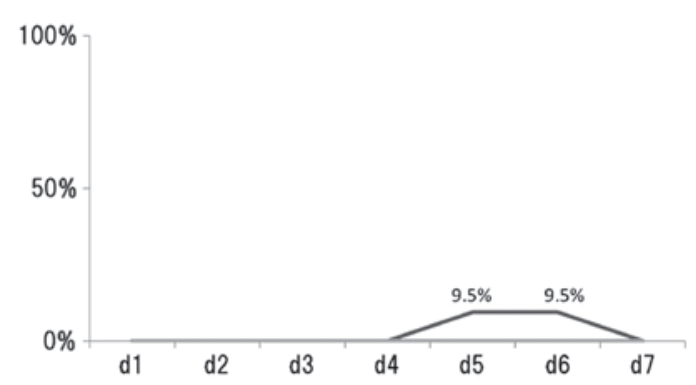

Figure 2. Percentage of patients reporting vomiting/day after chemotherapy treatment is shown. Upper line indicates the incidence of nausea in patients administered CDDP-regimens. No incidence of nausea was observed in patients administered CBDCA-regimens or those administered the nonplatinum regimen, respectively.

vomiting on days 5 and 6 . However, none of the patients treated with CBDCA-regimens and without platinum-regimens exhibited vomiting on days 1-7. 


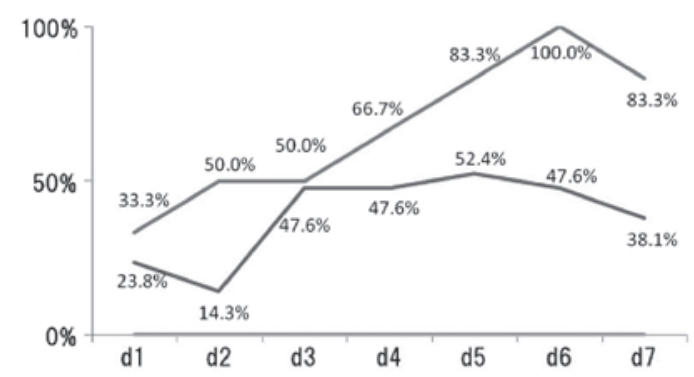

Figure 3. Percentage of patients reporting appetite loss/day after chemotherapy treatment is shown. Upper line indicates the incidence of nausea in patients administered CDDP-regimens. Middle line indicates the incidence of nausea in patients administered CBDCA-regimens. Lower line indicates the incidence of nausea in patients administered non-platinum regimen.

Other complications. Three of the most common complications were constipation, general fatigue and appetite loss. The incidence of these complications was $77.4,71.0$ and $67.7 \%$, respectively. The incidence of dysesthesia, stomatitis and diarrhea was $19.4,12.9$ and $12.9 \%$, respectively.

The percentage of patients reporting appetite loss/day following chemotherapy treatment was recorded (Fig. 3). In patients treated with CDDP-regimens, the incidence of appetite loss each day was: day $1,33.3 \%$; day $2,50 \%$; day $3,50 \%$; day 4 , $66.7 \%$; day $5,83.3 \%$; day $6,100 \%$ and day $7,83.3 \%$. In patients treated with CBDCA-regimens, the incidence of appetite loss each day was: day $1,23.8 \%$; day $2,14.3 \%$; day $3,47.6 \%$; day 4 , $47.6 \%$; day $5,52.4 \%$; day $6,47.6 \%$ and day $7,38.1 \%$.

\section{Discussion}

Recently, a new class of antiemetic agents has been developed. The NK-1 receptor antagonist aprepitant, when combined with 5-HT3 receptor antagonist and dexamethasone, are linked to a significant reduction in acute and delayed emesis in patients administered platinum-based regimens (12). Although CINV has been one of the most significant adverse events of chemotherapy, its impact appears to be decreased by metoclopramide, 5 -HT3 antagonists and corticosteroids $(13,14)$. Several studies have assessed the accuracy with which physicians and nurses perceive the control of nausea and emesis among their own patients. These studies concluded that these healthcare providers underestimate the incidence of the delayed nausea as well as emesis subsequent to HEC or MEC (15-17). Nevertheless, the majority of these studies were completed prior to accepting aprepitant as a standard treatment and introducing it into daily practice for the prevention of CINV (13-17). In their study, Majem et al (18) assessed control rates in their own practice following the introduction of aprepitant with a view to evaluate the accuracy with which physicians and nurses perceive the incidence of CINV (18). In our study, incidence rates of complications including CINV were assessed by pharmacists, who had no conflict of interests with any pharmaceutical companies. To the best of our best knowledge, this is the first study to evaluate incidence rates of complications including CINV assessed by pharmacists.

Even in the era of new antiemetic agents, a number of patients receiving CDDP-based regimens and those treated with CBDCA-based regimens continued to experience nausea, although $<10 \%$ of patients treated with CDDP-based regimens had vomiting. Prior to the current era of new antiemetics, healthcare providers, such as physicians and nurses overestimated the control of delayed nausea and emesis $(15,16)$. Previously, Majem et al (18) reported that the control rate of CINV was $66.7 \%$ in 95 patients (87\% administered HEC). They described that predictions of the control rate of CINV by healthcare providers were more accurate compared to those previously reported for HEC regimens with CDDP (18). In our patients, the control rate of CINV was $67.7 \%$, which was almost identical to the findings reported by Majem et al (18). In their study, Hilarius et al (19) reported that CINV was worse in women and in younger patients. In that study, $69 \%$ of the patients were female, with a mean age of 56 years (19). In our patients, there was a high percentage of male patients $(80.6 \%)$, with a mean age of 69 years.

In addition, the regulation of other complications, such as constipation, general fatigue and appetite loss remained poor, as observed in the present study. No promising drugs have been found for chemotherapy-induced general fatigue or for appetite loss. The precise mechanism of chemotherapy-induced general fatigue has yet to be elucidated, and its effective treatment has not been established at present. In chemotherapy-induced constipation, there may be several mechanisms in various antitumor drugs, and therefore, no decisive drug has been found for this complication. However, regarding appetite loss, a number of recent studies demonstrated that certain herbal medicines may modulate ghrelin, which is considered a pivotal signal to the brain to stimulate feeding (20). Rikkunshito is one of these promising herbal drugs for the control of appetite loss (21-23) and a clinical trial for this drug is currently being delineated.

There were certain limitations to this study, including the small sample size, possible selection bias and lack of a standardized antiemetic regimen. However, this study has shown that CDDP-regimen-induced CINV as well as constipation, general fatigue and appetite loss continue to be problems. These findings suggest that the current management of patients receiving chemotherapy repeatedly should carefully be considered. Additionally, the use of scales, such as pharmacist-reported outcome instruments assessing the impact of CINV on the daily function of patients and assessing frequency, severity and duration of postchemotherapy-related complications are likely to help physicians to better manage chemotherapy.

In conclusion, CINV as well as constipation, general fatigue and appetite loss continue to be problems for patients receiving chemotherapy. Their incidence is underestimated by physicians and nurses. A better assessment of the incidence of these chemotherapy-related complications by medical oncologists and physicians as well as medical staff, is essential for their adequate control.

\section{References}

1. Peters BG: An overview of chemotherapy toxicities. Top Hosp Pharm Manage 14: 59-88, 1994.

2. Van Ryckeghem F and Van Belle S: Management of chemotherapy-induced nausea and vomiting. Acta Clin Belg 65: 305-310, 2010.

3. Grunberg SM and Hesketh PJ: Control of chemotherapy-induced emesis. N Engl J Med 329: 1790-1796, 1993. 
4. Hickok JT, Roscoe JA, Morrow GR, King DK, Atkins JN and Fitch TR: Nausea and emesis remain significant problems of chemotherapy despite prophylaxis with 5-hydroxytryptamine-3 antiemetics: a University of Rochester James P. Wilmot Cancer Center Community Clinical Oncology Program Study of 360 cancer patients treated in the community. Cancer 97: 2880-2886, 2003.

5. Hesketh PJ, Grunberg SM, Gralla RJ, et al: Aprepitant Protocol 052 Study Group: The oral neurokinin-1 antagonist aprepitant for the prevention of chemotherapy-induced nausea and vomiting: a multinational, randomized, double-blind, placebo-controlled trial in patients receiving high-dose cisplatin - the Aprepitant Protocol 052 Study Group. J Clin Oncol 21: 4112-4119, 2003.

6. Warr DG, Grunberg SM and Gralla RJ, et al: The oral NK(1) antagonist aprepitant for the prevention of acute and delayed chemotherapy-induced nausea and vomiting: pooled data from 2 randomised, double-blind, placebo controlled trials. Eur J Cancer 41: 1278-1285, 2005.

7. Warr DG, Hesketh PJ, Gralla RJ, et al: Efficacy and tolerability of aprepitant for the prevention of chemotherapy-induced nausea and vomiting in patients with breast cancer after moderately emetogenic chemotherapy. J Clin Oncol 23: 2822-2830, 2005.

8. Osoba D: Health-related quality-of-life assessment in clinical trials of supportive care in oncology. Support Care Cancer 8 $84-88,2000$

9. Barford KL and D'Olimpio JT: Symptom management in geriatric oncology: practical treatment considerations and current challenges. Curr Treat Options Oncol 9: 204-214, 2008.

10. Stokman MA, Spijkervet FK, Boezen HM, Schouten JP, Roodenburg JL and de Vries EG: Preventive intervention possibilities in radiotherapy- and chemotherapy-induced oral mucositis: results of meta-analyses. J Dent Res 85: 690-700, 2006.

11. Park SB, Krishnan AV, Lin CS, Goldstein D, Friedlander M and Kiernan MC: Mechanisms underlying chemotherapy-induced neurotoxicity and the potential for neuroprotective strategies. Curr Med Chem 15: 3081-3094, 2008.

12. Navari RM, Reinhardt RR, Gralla RJ, et al: Reduction of cisplatin-induced emesis by a selective neurokinin-1-receptor antagonist. L-754, 030 Antiemetic Trials Group. N Engl J Med 340: 190-195, 1999.
13. Coates A,Abraham S, Kaye SB, et al: On the receiving end-patient perception of the side-effects of cancer chemotherapy. Eur J Cancer Clin Oncol 19: 203-208, 1983.

14. Griffin AM, Butow PN, Coates AS, et al: On the receiving end. $\mathrm{V}$ : patient perceptions of the side effects of cancer chemotherapy in 1993. Ann Oncol 7: 189-195, 1986.

15. Erazo Valle A, Wisniewski T, Figueroa Vadillo JI, Burke TA and Martinez Corona R: Incidence of chemotherapy-induced nausea and vomiting in Mexico: healthcare provider predictions versus observed. Curr Med Res Opin 22: 2403-2410, 2006.

16. Grunberg SM, Deuson RR, Mavros P, et al: Incidence of chemotherapy-induced nausea and emesis after modern antiemetics. Cancer 100: 2261-2268, 2004.

17. Liau CT, Chu NM, Liu HE, Deuson R, Lien J and Chen JS: Incidence of chemotherapy-induced nausea and vomiting in Taiwan: physicians' and nurses' estimation vs. patients' reported outcomes. Support Care Cancer 13: 277-286, 2005.

18. Majem M, Moreno ME, Calvo N, et al: Perception of healthcare providers versus patient reported incidence of chemotherapy-induced nausea and vomiting after the addition of NK-1 receptor antagonists. Support Care Cancer 19: 1983-1990, 2011.

19. Hilarius DL, Kloeg PH, van der Wall E, van den Heuvel JJ, Gundy CM and Aaronson NK: Chemotherapy-induced nausea and vomiting in daily clinical practice: a community hospital-based study. Support Care Cancer 20: 107-117, 2012

20. Yada T, Kohno D, Maejima Y, et al: Neurohormones, rikkunshito and hypothalamic neurons interactively control appetite and anorexia. Curr Pharm Des: May 23, 2012 (Epub ahead of print).

21. Takeda H, Sadakane C, Hattori T, et al: Rikkunshito, an herbal medicine, suppresses cisplatin-induced anorexia in rats via 5-HT2 receptor antagonism. Gastroenterology 134: 2004-2013, 2008.

22. Takeda H, Muto S, Nakagawa K, Ohnishi S and Asaka M: Rikkunshito and ghrelin secretion. Curr Pharm Des: May 23, 2012 (Epub ahead of print).

23. Cheng KC, Li YX and Cheng JT: The use of herbal medicine in cancer-related anorexia/cachexia treatment around the world. Curr Pharm Des: May 23, 2012 (Epub ahead of print). 